\title{
Linewidth of resonances in scanning tunneling spectroscopy
}

\author{
L. Jdira, ${ }^{1}$ K. Overgaag, ${ }^{2}$ R. Stiufiuc, ${ }^{3}$ B. Grandidier ${ }^{3}$ C. Delerue, ${ }^{3}$ S. Speller, ${ }^{1}$ and D. Vanmaekelbergh ${ }^{2}$ \\ ${ }^{1}$ Institute for Molecules and Materials, Radbound University Nijmegen, Toernooiveld 1, 6525 ED Nijmegen, The Netherlands \\ ${ }^{2}$ Debye Institute, Utrecht University, P.O. Box 80 000, 3508 TA Utrecht, The Netherlands \\ ${ }^{3}$ Département ISEN, Institut d'Electronique, de Microélectronique et de Nanotechnologie, \\ UMR CNRS 8520, 41 Boulevard Vauban, F-59046 Lille Cedex, France
}

(Received 5 September 2007; published 9 May 2008)

\begin{abstract}
Cryogenic scanning tunneling spectroscopy is increasingly used to study the electronic structure of adatoms, molecules, and semiconductor quantum dots. However, the width of the conductance resonances that indicate the energy levels is much larger than the thermal energy, and this is not well understood. Here, we present a comprehensive study of the line shape and width of the conductance resonances observed with small colloidal semiconductor quantum dots. Experimentally, the line shape and width are studied for CdSe quantum dots of different sizes, with nanocrystals being chemically or physically attached to the substrate. The influence of the temperature is studied from $5 \mathrm{~K}$ up to room temperature. We have also varied the set-point current via the tip-to-dot distance to study the effects of dissipative heating of the quantum dot. We present basic calculations of the effects of electron-phonon coupling, charge and dipole fluctuations in the close environment of the quantum dot, mechanical oscillations of the quantum dot in the tunneling junction, and internal heating by nonresonant electron transport. A comparison with the experimental results shows that electron-phonon coupling forms the main contribution to the line broadening for the lowest resonance. Fluctuations of the charge landscape around the quantum dot are most likely involved in an additional broadening. More importantly, these potential fluctuations wash out the vibronic structure of the line shape that should arise from electronphonon coupling. Our results show that in the case of semiconductor quantum dots, internal heating due to nonresonant electron transport is not important.
\end{abstract}

DOI: 10.1103/PhysRevB.77.205308

PACS number(s): 68.37.Ef, 61.46.Hk

\section{INTRODUCTION}

Scanning tunneling microscopy and spectroscopy (STS) are increasingly used to study the electronic structure of individual (ad)atoms, ${ }^{1-4}$ atom vacancies, ${ }^{5}$ molecules, ${ }^{6-8}$ clusters such as $C_{60}, 9,10$ and semiconductor quantum dots (QDs). ${ }^{11-14}$ The nano-object under study is generally attached to an atomically flat, conducting substrate, and the tip of the scanning tunneling microscope (STM) is placed above it. A tunneling current $I$ is measured as a function of the bias $V$ between the tip and the substrate. The peaks in the electrical conductance $d I / d V$ versus bias, i.e., the resonances in the tunneling spectrum, would reveal all energy levels of the nano-object that can mediate the current between the tip and substrate. Hence, tunneling spectroscopy offers a direct method to study the energy levels of individual nanosystems. It is generally recognized that the electronic coupling between the nanosystem under study and the substrate and tip electrodes should be weak. This ensures that the true eigenstates of the nano-object are measured, which are not broadened or shifted by the effects of electronic coupling. In many cases, a tunneling barrier between the substrate and the molecule has been used, such as a thin insulating layer of $\mathrm{NaCl}$ (Refs. 5 and 6) or $\mathrm{Al}_{2} \mathrm{O}_{3}, 15$ or a monolayer of organic molecules. ${ }^{11,16}$ The coupling to the tip electrode can be minimized by measuring at a sufficiently large tip-molecule distance, which is controlled by the set-point current. The study of the electronic structure of atoms, molecules, and quantum dots with STS has been very successful and is rapidly expanding.

The phenomena that are not well understood are the shape and width of the observed conduction peaks in the spectrum: molecules and quantum dots show broad resonances with widths in the $100 \mathrm{meV}$ range. ${ }^{11,12,17,18}$ This is about hundred times larger than the thermal energy at $5 \mathrm{~K}$, the temperature at which the measurements are usually carried out. It should be remarked that broadening due to the lifetime of the electron in the molecule or the quantum dot is unable to explain a linewidth in the $100 \mathrm{meV}$ range. Indeed, from the uncertainty relation, the broadening due to the dwell time of the electron through the QD-substrate tunnel junction cannot be higher than a few $\mu \mathrm{eV}$ for currents in the nanoampere range. Often, the conductance peak line shape is Gaussian,5,12 in other cases, a vibronic structure has been observed., ${ }^{4,7,19}$

In the present work, we consider the linewidth and shape of the conductance peaks of small colloidal semiconductor QDs. The electronic structure of QD has been studied in detail during the last decade,$^{20}$ and a remarkable understanding of the tunneling spectra (except for the linewidth) has been obtained on the basis of semiempirical pseudopotential and tight-binding models. ${ }^{17,21-23}$ We study the linewidth of the resonances as a function of the size of the QD, the applied temperature, and the magnitude of the tunneling current, with the latter being determined by the substrate/barrier/ QD/vacuum/tip geometry. Focusing on the peak associated with the electron ground state of CdSe QD, we observe a variation of the peak shape as well of its width as a function of the QD size. In order to determine the physics of line broadening, the differential conductance has been calculated considering several sources of broadening: coupling of the electron to phonon modes in the QD, fluctuations of charges in the surrounding of the QD, or displacements of the QD in the junction. We also investigate the effects of internal heating of the QD under study due to nonresonant electron trans- 
port. Comparison between the experimental and theoretical linewidths reveals that moderate electron-phonon coupling leading to polaron transport through the QD is the main cause of broadening of the conductance peaks. Fluctuations of the tunneling current cause a further broadening of the resonances and hinder the observation of the vibronic features of electron-phonon coupling.

\section{EXPERIMENTAL RESULTS}

CdSe QDs were synthesized according to the literature methods. ${ }^{24}$ Prior to their deposition onto a metallic substrate, the QDs were characterized by transmission electron microscopy (TEM) and optical spectroscopy to determine their shape, average size, and size dispersion. The dots were found to be nearly spherical and to have a size dispersion of $5 \%-$ $10 \%$. Their surface was passivated by organic ligands. For the STS experiments, five different sets of samples were prepared: (a) isolated CdSe QDs, with an average size of $3 \mathrm{~nm}$, linked to a gold surface via hexane dithiol molecules; (b) isolated CdSe QDs, with an average size of $5 \mathrm{~nm}$, linked to a gold surface via hexane dithiol molecules; (c) CdSe QDs, with an average size of $3.4 \mathrm{~nm}$, assembled on a highly oriented pyrolytic graphite (HOPG) surface; (d) CdSe QDs, with an average size of $6.1 \mathrm{~nm}$, assembled on a HOPG surface; and (e) CdSe QDs, with an average size of $6.1 \mathrm{~nm}$, assembled on a gold surface. Assembly by drop casting resulted in monolayer arrays of QDs, which proved to be very stable under STM conditions.

From each sample, 10-20 individual QDs were investigated by STS. The experiments were performed in three different setups: a home build cryogenic STM working at $4.5 \mathrm{~K}$ with cut Pt-Ir tips and two different low temperature STM (Omicron Nanotechnology) working between $4.8 \mathrm{~K}$ and room temperature in ultrahigh vacuum with $\mathrm{W}$ tips. With both tip and sample held at the same temperature $T$, tunneling spectra were acquired by placing the STM tip above the center of a dot and disconnecting the feedback loop. The tunneling current was measured as a function of the tipsubstrate potential difference $V$. The tunneling differential conductance $G(V)=d I / d V$ was obtained either by digitally filtering and differentiating the experimental $I-V$ curves or by measuring the dynamic differential conductance by using a lock-in amplifier. Acquiring tens of scans above a given QD, we found that the $I-V$ curves were reproducible. Discrete shifts of the curves along the voltage axis can sometimes occur within a set of spectra acquired with the same feedback conditions. The reason for this could be the trapping of a charge in the surrounding of the dot that acts as a local gate and leads to a shift of the measured spectrum. ${ }^{25,26} \mathrm{As}$ a result, only those curves that are shifted by less than $\pm 4 \mathrm{mV}$ were averaged together for a given dot. In most cases, a spectrum corresponds to the average of a few tens of curves allowing a good signal to noise ratio.

Figure 1 shows typical tunneling spectra obtained on isolated CdSe QD linked to a gold surface via hexane dithiol molecules. The spectra, which are acquired at low current set points, have been discussed in a previous study. ${ }^{12}$ With such feedback conditions, the tunneling rate between the tip and
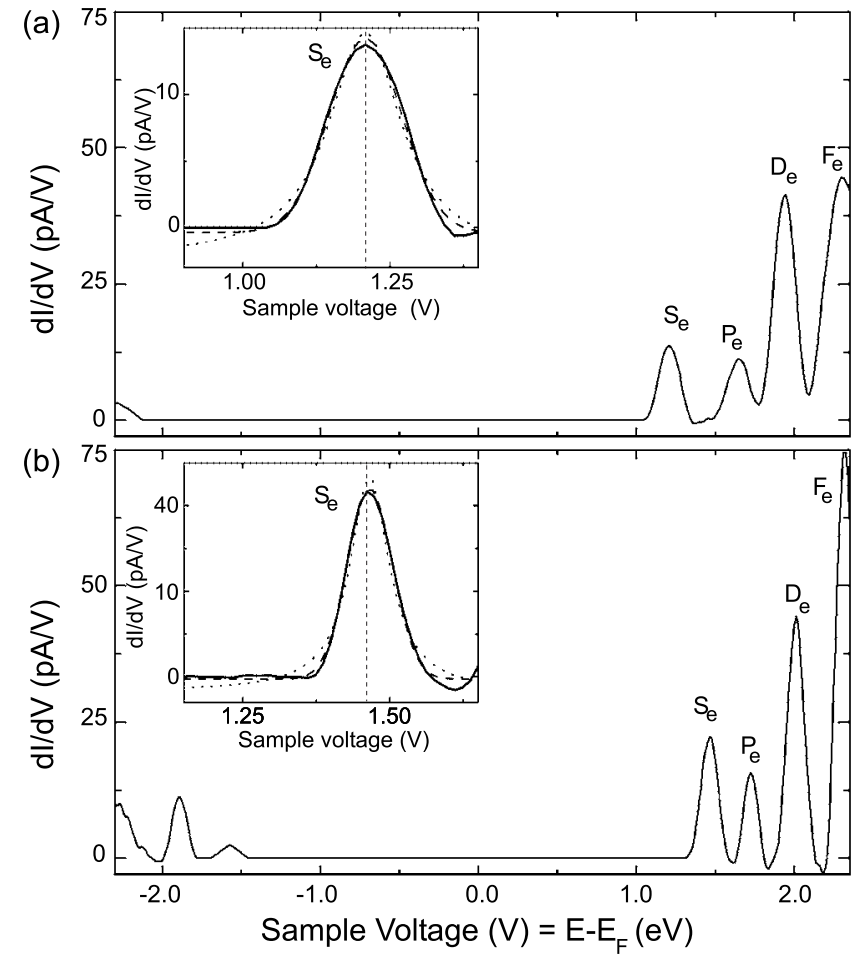

FIG. 1. Differential conductance spectra for isolated CdSe QDs in the shell-tunneling regime for two samples with TEM, which determined core sizes of (a) 3.0 and (b) $5.0 \mathrm{~nm}$. Both spectra were acquired at a temperature of $4.9 \mathrm{~K}$ with feedback parameters of $V$ $=2.5 \mathrm{~V}$ and $I_{s}=20 \mathrm{pA}$. The peaks in the conduction band are labeled $S_{e}, P_{e}, D_{e}$, and $F_{e}$. Inset: enlarged views of the $S_{e}$ peak. The vertical dotted line indicates the peak maximum position. The $S_{e}$ peaks were fitted with a Gaussian (dashed line) and a Lorentzian (dotted line) line shape.

the QD is smaller than the one between the QD and the gold substrate. Therefore, the series of peaks observed at positive sample bias correspond to the single-particle energy levels of the QD conduction band (shell-tunneling regime), which are labeled by the symmetry of the wave functions $S_{e}, P_{e}$, and $D_{e} \cdot{ }^{12}$ A detailed observation of the $S_{e}$ peaks indicates that the line shapes are better fitted by a Gaussian than a Lorentzian function (Fig. 1). Interestingly, the symmetry of the peak changes with the dot diameter. The dotted line running through the maximum of the peaks in the inset of Fig. 1 shows that the $S_{e}$ peak for a QD size of $3 \mathrm{~nm}$ has a symmetric shape and that a slight asymmetry becomes visible for the bigger QD. We also note that the full widths at half maximum (FWHMs) are 151 and $96 \mathrm{meV}$ for the QDs with diameters of 3 and $5 \mathrm{~nm}$, respectively.

Figure 2 shows the tunneling spectra obtained on a bigger QD with a diameter of $6.1 \mathrm{~nm}$ for two different set point currents. Although the measurements were performed on a QD positioned in the middle of an array assembled on a gold substrate, the spectra exhibit a zero-conductivity gap and a series of peaks in the conduction band very similar to the ones observed in Fig. 1. Such apparent gap and peak separation suggest that the electronic interaction between the dots is negligible and does not lead to an additional broadening of the $S_{e}$ peak as observed in the case of PbSe or InAs QDs. ${ }^{27,28}$ 


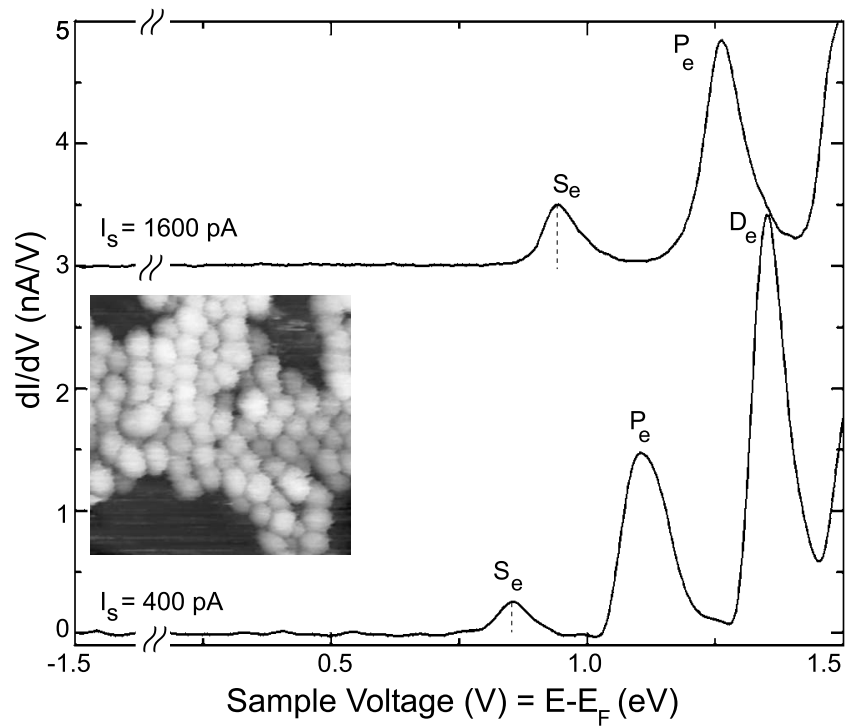

FIG. 2. Differential conductance spectra for a CdSe QD on gold in shell-tunneling regime for two different set point tunneling currents $I_{s}$. The dot belongs to an array of dots with a TEM determined core size of $6.1 \mathrm{~nm}$. The peaks in the conduction band are labeled $S_{e}, P_{e}$, and $D_{e}$. The vertical dotted segments indicate the $S_{e}$ peak maximum position.

Compared to isolated QDs, the asymmetry of the peak shape is more pronounced in the present results.

We remark that the spectra of CdSe QDs in an array are very stable in a broad current range and always reflect the single-particle energy levels (i.e., shell-tunneling conditions). This is in contrast with isolated QDs where a transition to shell filling is observed at higher current. Whereas electrons in an isolated QD can only escape by tunneling to an electronic state of the gold substrate, the transfer rate for an electron escaping out of a QD in an array is expected to be higher. As a result, filling the $S_{e}$ orbital with a second electron, which would cause the occurrence of an additional charging peak between the $S_{e}$ and $P_{e}$ peaks, is rarely seen for set point currents below a few nanoamperes (only in $2 \%$ of the spectra).

As in tunneling experiments, the applied voltage between the tip and the gold surface does not completely drop across the tip-QD junction; the measured zero-conductivity gap is always larger than the quasiparticle gap of the QD by a factor $1 / \eta$, where $\eta$, the so-called level arm, corresponds to the ratio between the potential drop in the tip-QD junction and the applied voltage. ${ }^{17,20-22}$ Similar to the quasiparticle gap, the widths of the peaks associated with the quantized states of the QD are also broadened by the factor $1 / \eta$. Although $\eta$ may be obtained by solving the Poisson equation, ${ }^{22,27}$ we prefer to determine $\eta$ from the ratio between the theoretical $S_{e}-P_{e}$ peak separation and the measured $S_{e}-P_{e}$ peak separation. Based on the theoretical $S_{e}-P_{e}$ transitions of CdSe QDs calculated in Ref. 29, which were quite consistent with the measured optical $S_{e}-P_{e}$ transitions, we obtain $\eta$ of 0.84 and 0.66 for set point currents of 400 and $1600 \mathrm{pA}$, respectively. Multiplying the FWHM of both $S_{e}$ peaks by the related $\eta$ factor finally yields a normalized FWHM of $54 \mathrm{meV}$ for both peaks. Applying the same normalization to the width of the
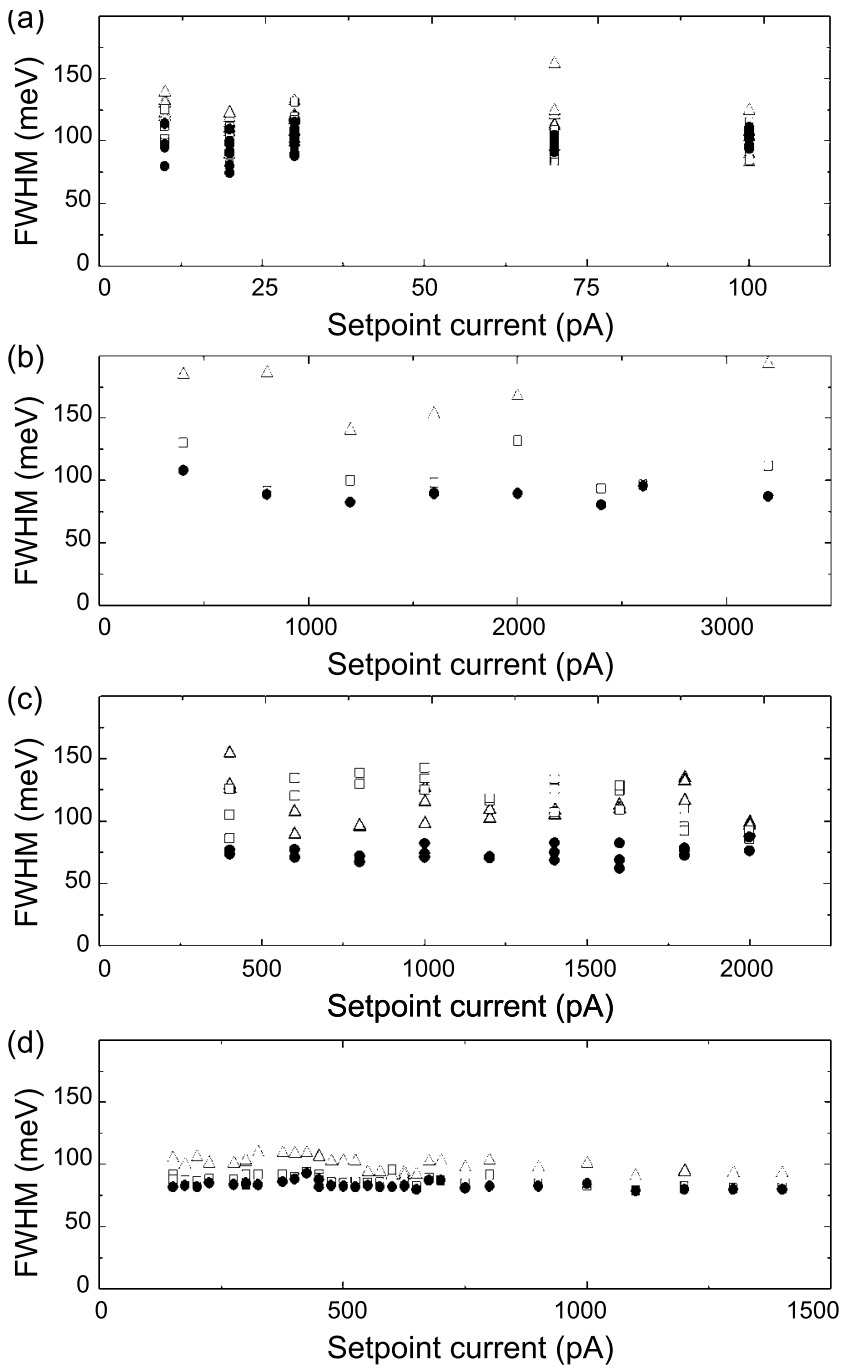

FIG. 3. Full width at half maximum (FWHM) of the $S_{e}(\bullet), P_{e}$ $(\square)$, and $D_{e}(\triangle)$ peaks, which are measured as a function of the set point tunneling current for (a) an isolated CdSe dot linked to the hexanedithiol/Au surface (averaged TEM core size of $3 \mathrm{~nm}$ ), (b) a dot in an array of CdSe dots on HOPG (averaged TEM core size of $3.4 \mathrm{~nm}$ ), (c) a dot in an array of CdSe dots on HOPG (averaged TEM core size of $6.1 \mathrm{~nm}$ ), and (d) a dot in an array of CdSe dots on $\mathrm{Au}$ (averaged TEM core size of $6.1 \mathrm{~nm}$ ).

$S_{e}$ peaks visible in Fig. 1, we find normalized FWHMs of 146 and $86 \mathrm{meV}$ for the QDs with diameters of 3 and $5 \mathrm{~nm}$, respectively, suggesting that the FWHMs decrease with increasing QD size.

We investigated if the width of the peaks increases with increasing tunneling current. Figure 3 shows the corrected FWHMs of the $S_{e}, P_{e}$, and $D_{e}$ peaks for four CdSe QDs on different substrates in a set point current range corresponding to the shell-tunneling regime. As already mentioned above, the set point current can be varied in a much wider range for the QDs in an array, without observing the occurrence of additional charging peaks in the spectra. Remarkably, no matter what the environment of the QDs and their sizes are, the FWHM of the $S_{e}$ peak is independent of the applied current, the smallest QDs yielding the larger FWHM. We also note that the FWHM tends to increase for the QD elec- 


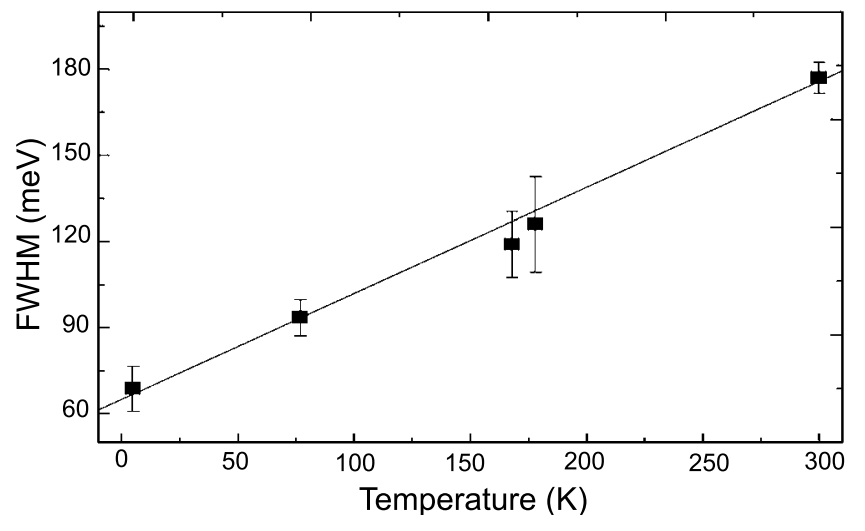

FIG. 4. FWHM of the $S_{e}$ peak, which is measured on CdSe dots, with a TEM determined core size of $6.1 \mathrm{~nm}$, as a function of temperature. The dots belong to array assembled on the gold and HOPG surfaces. The FWHM is normalized by the ratio between the theoretical (from Ref. 29) and measured $S_{e}-P_{e}$ peak separation. Line: fit showing that the FWHM linearly varies with a slope of $0.37 \mathrm{meV} / \mathrm{K}$.

tronic orbitals with higher spatial extension and degeneracy.

The same behavior was found at temperatures of up to $300 \mathrm{~K}$ for QDs with a size of $6.1 \mathrm{~nm}$ : the FWHM is independent of the set point current in the shell-tunneling regime. Although it was not possible to measure a complete temperature series on the same QD, due to experimental requirement of very good temperature stability, Fig. 4 shows the average FWHM of the $S_{e}$ peaks, which is acquired for several QDs and normalized by the level arm, as a function of the temperature. In the temperature range of 5-300 K, the FWHM clearly linearly increases with the applied temperature.

\section{THEORETICAL RESULTS}

\section{A. Current through a level coupled to a local vibration mode}

Now, we discuss the line shape of a conductance peak corresponding to the injection of an electron from the tip to a nondegenerate state of the QD at the energy $\varepsilon_{0}$. We assume that $G(V)$ would be a Dirac function in the absence of electron-vibration coupling, i.e., $G(V) \propto \delta\left(e V-\varepsilon_{0}\right)$. We first consider the simplest case where an electron on the electronic state interacts with a single vibrational mode of energy $\hbar \omega$ localized on the QD. We neglect the effect of the vibrations in the leads (for justifications, see, for example, Refs. 30 and 31). The electron-vibration coupling is characterized by the Huang-Rhys ${ }^{32}$ factor $S$ such that $\lambda=S \hbar \omega$ is the reorganization (relaxation) energy (or polaron energy shift) due to injection of the electron into the QD ( $S$ is also often denoted as $g$ and $\lambda$ also corresponds to the Frank-Condon shift $d_{\mathrm{FC}}$ in optical experiments ${ }^{33}$ ). The probability per unit time for the transfer of an electron from the tip to the QD is obtained from Fermi's golden rule, ${ }^{20,34}$

$$
\begin{aligned}
W= & \frac{2 \pi}{\hbar}\left|\beta_{i f}\right|^{2} \sum_{n_{i}} p\left(n_{i}\right) \sum_{n_{f}}\left|\left\langle\chi_{n_{f}}\left(Q-Q_{f}^{0}\right) \mid \chi_{n_{i}}\left(Q-Q_{i}^{0}\right)\right\rangle\right|^{2} \\
& \times \delta\left(E_{i, n_{i}}-E_{f, n_{f}}\right),
\end{aligned}
$$

where $i$ and $f$ denote the initial and final states of energy $E_{i, n_{i}}$ and $E_{f, n_{f}}$, respectively, $\beta_{i f}$ is the tunneling matrix element between electronic states (supposed here to be a constant), and $Q$ is the normal coordinate of the vibrational mode. $\left|\chi_{n_{f}}\left(Q-Q_{f}^{0}\right)\right\rangle$ and $\left|\chi_{n_{i}}\left(Q-Q_{i}^{0}\right)\right\rangle$ are the eigenstates of the harmonic oscillators centered at the positions $Q_{f}^{0}$ and $Q_{i}^{0}$, respectively. $Q_{f}^{0}$ differs from $Q_{i}^{0}$ due to the lattice relaxation induced by electron injection $\left(Q_{i}^{0}-Q_{f}^{0}=\sqrt{2 S \hbar / \omega}\right) \cdot{ }^{20,33} p\left(n_{i}\right)$ is the probability to have $n_{i}$ quanta of vibrations in the initial state at the temperature $T$. The calculation in Eq. (1) of the overlaps between displaced oscillators can be found in many references leading to ${ }^{20,32-36}$

$$
G(V) \propto \sum_{p} W_{p} \delta\left(e V-\varepsilon_{0}-p \hbar \omega\right),
$$

with

$$
W_{p}=\exp \left[\frac{p \hbar \omega}{2 k T}-S \operatorname{coth}\left(\frac{\hbar \omega}{2 k T}\right)\right] I_{p}\left\{\frac{S}{\sinh (\hbar \omega / 2 k T)}\right\},
$$

where $I_{p}$ is a modified Bessel function. Similar equations can be derived from Refs. 30 and 31. Positive (negative) values of $p$ correspond to the emission (absorption) of vibrations. In the strong coupling limit $(S \gg 1)$, the expression of $W_{p}$ becomes $(|p-S| \ll S)$

$$
W_{p} \approx \frac{\exp \left(-\frac{(p-S)^{2}}{2 S \operatorname{coth}(\hbar \omega / 2 k T)}\right)}{\sqrt{2 \pi S \operatorname{coth}(\hbar \omega / 2 k T)}},
$$

which shows that $G(V)$ is a series of peaks whose envelope is a Gaussian centered on $p=S$. The FWHM of the Gaussian is given by ${ }^{20,31}$

$$
\Delta E=\sqrt{8 \ln (2) \lambda \hbar \omega \operatorname{coth}(\hbar \omega / 2 k T)} .
$$

From this expression, two opposite situations clearly emerge. When $k T \ll \hbar \omega[\operatorname{coth}(\hbar \omega / 2 k T) \approx 1], \Delta E$ is independent of the temperature and is only determined by the electron-vibration coupling strength $(S)$ and the phonon energy. Recent STS experiments on a dangling bond state at a Si surface have shown results belonging to this category. ${ }^{4}$ It is also interesting to note that when the temperature goes to zero, $W_{p}$ is also exactly given by $(p \geq 0)$,

$$
W_{p}=\frac{S^{p}}{p !} e^{-S} .
$$

In the opposite situation $(k T \gg \hbar \omega)$ that corresponds to the classical limit $[\operatorname{coth}(\hbar \omega / 2 k T) \approx 2 k T / \hbar \omega], \Delta E$ behaves as $\sqrt{\lambda k T}$ and the Gaussian line shape simply arises from the thermal fluctuation of the oscillator. When the electron is coupled to several vibrational modes, the full line shape is given by the convolution of all the line shapes corresponding to the coupling to each individual mode. ${ }^{20,35,36}$ This may lead to a strong broadening such that the identification of the individual vibronic peaks may become difficult. However, even in that case, the Gaussian line shape can be recovered in different limits of strong electron-phonon coupling or at high temperature limit. ${ }^{33}$ 


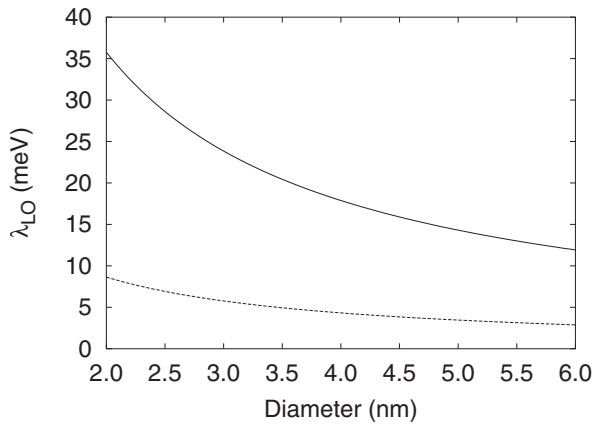

FIG. 5. Polaron energy shift (relaxation energy) $\lambda_{\mathrm{LO}}$ induced by the coupling to LO phonons [Eq. (7)] as a function of the diameter of the quantum dot. For CdSe (solid line): $\varepsilon_{\text {in }}(0)=9.3, \varepsilon_{\text {in }}(\infty)=6.1$, $\hbar \omega_{\mathrm{LO}}=26 \mathrm{meV}$, and $R_{P}=3.3 \mathrm{~nm}$. For InAs (dashed line): $\varepsilon_{\mathrm{in}}(0)$ $=15.1, \varepsilon_{\text {in }}(\infty)=12.3, \hbar \omega_{\mathrm{LO}}=30 \mathrm{meV}$, and $R_{P}=7.4 \mathrm{~nm}$.

In Sec. III B, we consider the coupling to longitudinal optical (LO) phonons because we will see that it substantially contributes to the line shape. Other possible mechanisms for the broadening are discussed in Appendices A-C.

\section{B. Coupling to longitudinal optical phonons}

In polar crystalline materials, the coupling of an electron to LO phonons can strongly influence the electronic and optical properties. In the case of QDs, there exist bulk-type and interface-type phonons which may be involved in the formation of polarons. In spherical QDs, extensive work shows that coupling to interface-type LO phonons is weak and that bulk-type phonons play the dominant role in the polaron energy shift. ${ }^{20,37-40}$ When the QD radius $R$ is smaller than the polaron radius $R_{p}=\sqrt{\hbar /\left(2 m^{*} \omega_{\mathrm{LO}}\right)}$, where $m^{*}$ is the effective mass, the polaron energy shift can be calculated using the adiabatic approximation giving for an electron in an $S$ state, ${ }^{20,37-40}$

$$
\lambda_{\mathrm{LO}} \approx 0.39 \times \frac{e^{2}}{R}\left(\frac{1}{\epsilon_{\text {in }}(\infty)}-\frac{1}{\epsilon_{\text {in }}(0)}\right),
$$

where $\epsilon_{\text {in }}(0)$ and $\epsilon_{\text {in }}(\infty)$ are the static and the high-frequency dielectric constants of the QD material, respectively. Figure 5 presents the variation of the relaxation energy $\lambda_{\mathrm{LO}}$ with $R$ for CdSe and InAs QDs. $\lambda_{\mathrm{LO}}$ is much larger in CdSe than in InAs because it is a more ionic material. Even if $\lambda_{\text {LO }}$ reaches substantial values at small radius, the strong coupling regime $\left(S=\lambda_{\mathrm{LO}} / \hbar \omega_{\mathrm{LO}} \gg 1\right)$ is almost never obtained.

In Fig. 6, we plot the differential conductance calculated by using Eqs. (2) and (6) at $0 \mathrm{~K}$. We have considered an electron coupled to LO modes and the results are shown for CdSe QDs of different diameter (2 or $4 \mathrm{~nm}$ ). The spectra (solid lines) are sums of peaks whose envelope is strongly asymmetric because we are in an intermediate coupling regime $(S \lesssim 1)$.

\section{DISCUSSION}

In the following, we discuss the origins of the $S_{e}$ peak broadening and its dependence on external parameters. We first consider to which extent nonresonant tunneling can lead

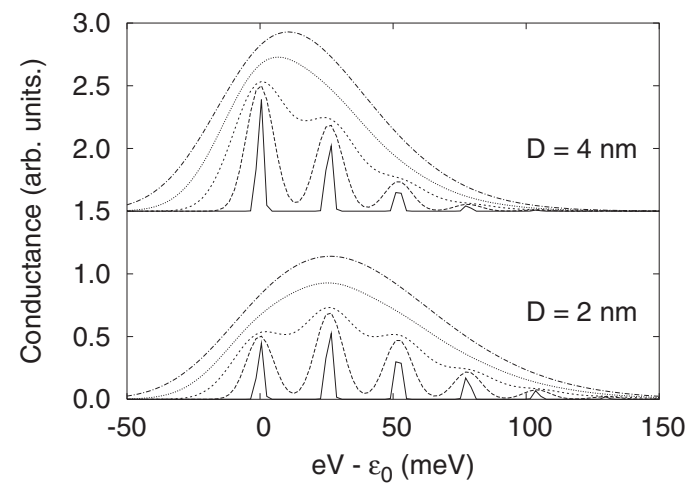

FIG. 6. Tunneling conductance at $T=0 \mathrm{~K}$ versus the energy $\left(e V-\varepsilon_{0}\right)$ for an electron in an $S$ state of a CdSe QD coupled to LO phonons. Results are shown for two QD diameters and are calculated using Eqs. (2) and (6) in which each Dirac function is broadened by a Gaussian. The different curves correspond to different widths of the Gaussians (FWHM=2.4, 12, 24, 36, and $48 \mathrm{meV}$ ).

to an increased temperature of the QD. The physical model is presented in Appendix D. It is shown that nonresonant tunneling can lead to an almost linear increase in the dot temperature with the current if the heat dissipation is insufficient. It follows from Eqs. (5) and (D2) that the FWHM should be proportional to the square root of the tunneling current. However, Fig. 3 shows that in our experiments, the FWHM does not depend on the tunneling current. Hence, heat dissipation is effective and the dot temperature equals the temperature in the cryostat. This also means that at $5 \mathrm{~K}$, the thermal energy is much smaller than the LO phonon mode.

In this situation, the shape of the $S_{e}$ peak should depend on the strength of the electron-phonon coupling: for strong electron-phonon coupling, the peak should exhibit a symmetric Gaussian shape, whereas in the intermediate regime, the Gaussian line shape should be asymmetric, as shown in Fig. 6. Such variation of the line shape is consistent with the experimental observations of Figs. 1 and 2. Indeed, for the QD with the smallest size, where the electron confinement is the highest and thus the electron-phonon coupling is the strongest, the $S_{e}$ peak was best fitted by a Gaussian. However, when the QD size increases, the theoretical line shape becomes more asymmetric (Fig. 6) and the $S_{e}$ peak can no longer be fitted by a single Gaussian, as shown for the lower fit in Fig. 7 in the case of a QD with a $6.1 \mathrm{~nm}$ diameter. Instead, by considering that the line shape is given by a series of peaks whose amplitude is determined by the coupling to LO phonons $\lambda_{\mathrm{LO}}$ given by Eq. (7), we are able to fit the $S_{e}$ peak, when each vibronic peak is broadened by a Gaussian with a FWHM of $36.9 \mathrm{meV}$ (upper curve in Fig. 7). The broadening of the vibronics that we use here in the fits must have a physical origin too; this will be discussed below.

We discuss in Appendices A-C that the coupling to acoustic phonons or the dielectric response of the polarizable organic capped layer leads to further broadening. However, with predicted FWHMs of 2 (Appendix A) and $9 \mathrm{meV}$ (Appendix B), the linewidth cannot be fully explained by such effects for QDs with a $6.1 \mathrm{~nm}$ diameter. Mechanical oscillations of the QD in the junction are also a possible cause of 


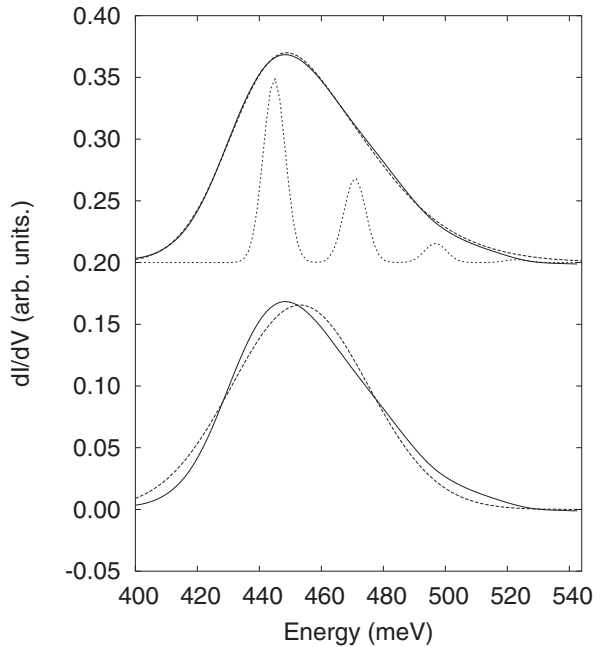

FIG. 7. Comparison between experimental (continuous line) and calculated (dotted lines) differential conductances for an electron in a $S_{e}$ state of a CdSe QD, with a diameter of $6.1 \mathrm{~nm}$. The lower theoretical curve is a simple Gaussian fitted to the experimental conductance peak. The upper ones are obtained for an electron coupled to the LO phonon mode $\left(\hbar \omega_{\mathrm{LO}}=26 \mathrm{meV}\right)$ and correspond to a series of vibronic peaks broadened by Gaussians with widths of 8.3 or $36.9 \mathrm{meV}$.

broadening that we analyze in Appendix C. However, since similar linewidths are obtained for the five sets of samples, which are independent of the nature of the QD-substrate coupling and of the tip-QD distance, we conclude that it cannot explain our observations either.

Therefore, there must be another origin of line broadening. In the following, we show that charge fluctuations in the environment of the dot in the tip/dot/substrate junction could play an important role. Such fluctuations have been invoked to explain the spectral diffusion of the photoluminescence of QDs. ${ }^{41-44}$ The width of the STS peaks is then related to the variations of the electron energy in the QD due to the displacements of external charges. To estimate this effect, it is interesting to consider the charging energy $U$ required to inject a second electron on the same shell of a QD. This energy has been measured by shell-filling spectroscopy and, for diameters of $3 \mathrm{~nm}$, values of $\approx 135$ and $\approx 250 \mathrm{meV}$ have been reported for $\mathrm{CdSe}$ (Ref. 12) and InAs QDs, ${ }^{11}$ respectively. For charges at the surface or outside the QDs, the variations in the electronic energy are expected to be smaller. We conclude, however, that charge fluctuations may contribute to broadening.

To get a deeper insight into the influence of charge fluctuations, the variations of the tunneling current as a function of time were thus acquired at the voltage $V_{S_{e}}$ that corresponds to the position of the maximum of the $S_{e}$ peak. To ensure that the tunneling current was really measured at the energy of the $S_{e}$ peak maximum, the voltage was first ramped down to zero and ramped back up to $V_{S}$, where the voltage was then kept constant for duration of tens of seconds. During this period of time, the fluctuations of the current were acquired with a sampling rate of $10 \mu$ s and then recorded every $5.12 \mathrm{~ms}$. Figure 8 shows the variations of the current intensity at the voltage $V_{S_{e}}$ for a QD with a $6.1 \mathrm{~nm}$ diameter.
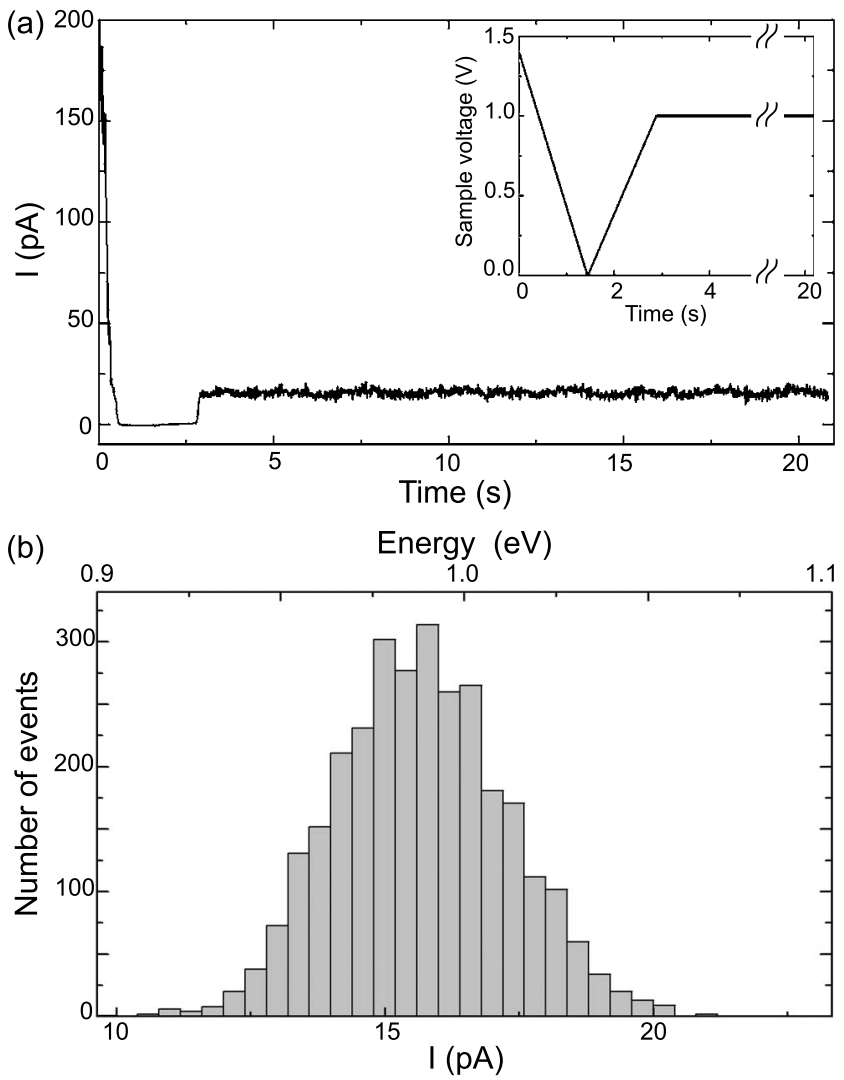

FIG. 8. (a) Fluctuations of the tunneling current as a function of time for a voltage corresponding to the peak maximum position of the $S_{e}$ orbital. Inset: voltage contour used to ensure that the measurement of the tunneling current occurs at the peak maximum position of $S_{e}$ orbital. (b) Histogram showing the distribution of the tunneling current fluctuations. The current intensity is translated into energy on the upper horizontal axis.

By using the left part of the $I-V$ curve, we can convert the tunneling current variation into energy and we find a FWHM of $56 \mathrm{meV}$ for the distribution of the fluctuations, which is in good agreement with the range of FWHMs experimentally found from the differential conductance $S_{e}$ peaks.

From this measurement, it is clear that fluctuations of the current contribute to the linewidth of the $S_{e}$ peak and impede the observation of the vibronic peaks in tunneling spectroscopic experiments of CdSe QDs. Redistribution of charges at the surface of QDs or in the vicinity of QDs has been invoked to explain spectral fluctuations observed in optical spectroscopy of colloidal QDs, such as intermittency fluorescence, ${ }^{41-43}$ and such phenomenon is likely to be enhanced by the high electrical field applied in the tunneling junction. ${ }^{45}$ More recently, the movement of surface charges was confirmed through optical spectroscopic measurements performed on nanorod consisting of a CdSe core and an elongated $\mathrm{CdS}$ shell. ${ }^{44}$

Interestingly, in this latter study, a linear dependence of the broadening as a function of the temperature was observed, which is similar to the one found in Fig. 4, where we obtain a slope of $0.37 \mathrm{meV} / \mathrm{K}$ for the linear fit. Such a coefficient is quite close to the value of $0.30 \mathrm{meV} / \mathrm{K}$ expected for a broadening induced by the spread of the electron energy 
distribution. As a result, when the temperature increases, the evolution of the Fermi-Dirac distribution of the electrons in the tip is likely to hide all other possible mechanisms involved in the spectral broadening.

Finally, the FWHMs of the $P_{e}$ and $D_{e}$ peaks are found to be larger than the $S_{e}$ peaks, as indicated in Fig. 3. These levels are assumed to be degenerate. However, due to an intrinsic crystal electric field or the field over the tip/dot/ substrate junction, the degeneracy of the $P_{e}$ and $D_{e}$ levels could be lift off, which results in an additional broadening of these resonances.

\section{CONCLUSIONS}

In conclusion, we have investigated the linewidth of the resonance associated with the single-particle $S_{e}$ level in the conduction band of CdSe QDs. By studying QDs with different sizes, by using different set point currents and measurement temperatures, we show that the coupling of LO phonons to the tunneling electron largely contributes to the broadening of the peaks in the tunneling spectra, without rising the internal temperature of the QD to a significant extent. The coupling to LO phonons also explains the asymmetric line shape observed for nanocrystals with a large diameter $(6.1 \mathrm{~nm})$. We show that the fluctuations of the current caused by fluctuations of the environment lead to an additional broadening, which explains why LO phonon replica of the peaks are not observable in these conditions. If these fluctuations could be reduced, we predict that the effects related to the coupling to acoustic phonons or mechanical vibrations of the QDs could be seen in some cases.

A number of the mechanisms that we have discussed here for nanocrystal QDs could also be important in the tunneling spectroscopy of molecules. For instance, vibrational modes have been observed in a number of cases ${ }^{7,8}$ while in other cases, only one broad resonance is observed. ${ }^{6}$ In the latter case, the vibrations are washed out, and the mechanisms that we have discussed here, e.g., fluctuations in the charge landscape and dipolar environment (Appendix B), oscillations of the molecule in the junction (Appendix $\mathrm{C}$ ), and even heating of the molecule due to nonresonant tunneling could be the origins for this.

\section{ACKNOWLEDGMENTS}

This work was supported by the Stichting voor Fundamenteel Onderzoek der Materie FOM which is financially supported by the Netherlands Organization for Scientific Research NWO, and the Nanotechnology network in the Netherlands NanoNed, the Dutch nanotechnology program of the Ministry of Economic Affairs.

\section{APPENDIX A: BROADENING INDUCED BY THE COUPLING TO ACOUSTIC PHONONS}

An electron injected into a QD also couples to acoustic phonons. Simple models of coupling to acoustic modes can be found in literature showing that the relaxation energy $\lambda$ very quickly decreases with QD size, like $1 / R^{3} \cdot{ }^{20,46-48}$ For CdSe QDs, $\lambda \approx 16 \mathrm{meV}$ can be estimated for $R=1.1 \mathrm{~nm},{ }^{46,47}$ which gives $\lambda \approx 6 \mathrm{meV}$ for $R=1.5 \mathrm{~nm}$ and $\lambda \approx 2.7 \mathrm{meV}$ for $R=2 \mathrm{~nm}$. Similar values are predicted by using the methods described in Ref. 20. Thus, the coupling to acoustic modes certainly contributes to the broadening of the STS peaks but only in small QDs. However, we have to take care that these models are based on several approximations, and recent density functional theory (DFT) calculations have shown that they underestimate the relaxation energy in small Si QDs. ${ }^{49}$ DFT calculations of the structural relaxation in charged CdSe QDs should be of high interest in this context.

\section{APPENDIX B: BROADENING INDUCED BY THE POLARIZATION OF THE ENVIRONMENT}

Colloidal QDs are surrounded by entities such as polar molecules which, in response to the electric field induced by the injection of an electron in the QD, may reorient themselves or have some structural relaxation leading to a polarization field (i.e., formation of an organic polaron). Following the model proposed by Marcus ${ }^{50}$ and many others, thermal fluctuations of the environment couple to the electron that is then allowed to tunnel from the tip to the QD nonresonantly, with the difference in energy between the initial and final states being provided by the environment. Such a mechanism was invoked by Brus ${ }^{51}$ to explain the higher conductance of porous silicon in the presence of a polar liquid, where the electrons tunnel from dot to dot. In the likely situation where $k T$ is higher than the typical vibration energies (for comparison, $\approx 0.1 \mathrm{meV}$ for water at room temperature), the broadening is Gaussian. To calculate the electron molecular vibration coupling, we consider a simple model in which the QD is surrounded by a dielectric layer of thickness $\delta R$ and of static (dynamical) dielectric constant $\varepsilon_{\text {out }}(0)$ $\left[\varepsilon_{\text {out }}(\infty)\right]$. The relaxation energy is given by

$$
\lambda_{p}=\left(\frac{1}{2} \int_{\Omega} \rho(r) V(r) d r\right)_{\infty}-\left(\frac{1}{2} \int_{\Omega} \rho(r) V(r) d r\right)_{0},
$$

where $V(r)$ is the electrostatic potential induced by the $S_{e}$ electronic density $\rho(r)$ and $\Omega$ is the QD volume. The factor $1 / 2$ comes from the fact that it is a self-energy. We make the difference between the high-frequency limit $(\infty)$ and the static one (0) to keep only the response of the vibrations. By using the Gauss theorem, we are left with

$$
\lambda_{p}=\frac{e^{2}}{2}\left(\frac{1}{\varepsilon_{\text {out }}(\infty)}-\frac{1}{\varepsilon_{\text {out }}(0)}\right)\left(\frac{1}{R}-\frac{1}{R+\delta R}\right),
$$

which, as long as $\rho(r)$ has the spherical symmetry, neither depends on the semiconductor dielectric constant $\varepsilon_{\text {in }}$ nor on the expression of $\rho(r)$. Figure 9 presents the variation of $\lambda_{p}$ with size for $\delta R=0.5 \mathrm{~nm}$, with $\varepsilon_{\text {out }}(0)=20$ and $\varepsilon_{\text {out }}(\infty)=2$. The FWHM of the STS peaks induced by this coupling is also shown for two temperatures, 5 and $30 \mathrm{~K}$ [Eq. (5) in the limit $k T \gg \hbar \omega]$. We see that the calculated FWHM can be substantial but, with a value of $9 \mathrm{meV}$, is not sufficient to explain the observed linewidths for diameters of $6.1 \mathrm{~nm}$. 


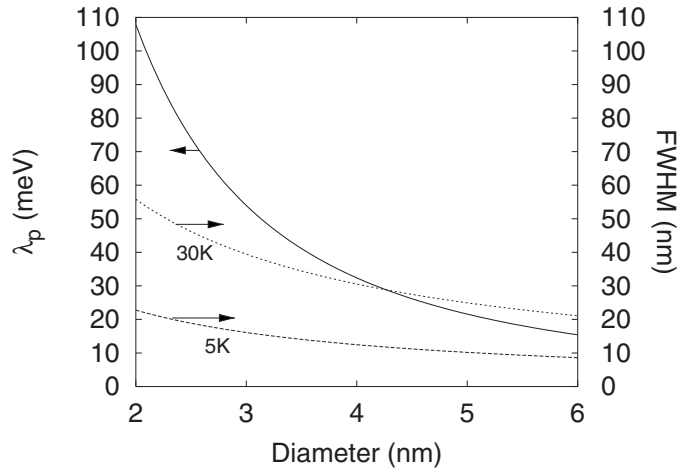

FIG. 9. Solid line: Relaxation energy $\lambda_{p}$ of an electron in an $S$ state induced by the dielectric response of a $0.5 \mathrm{~nm}$ thick polarizable layer around the QD versus its diameter $\left(\varepsilon_{\text {out }}(0)=20\right.$ and $\left.\varepsilon_{\text {out }}(\infty)=2\right)$. FWHM of the STS peak induced by this relaxation calculated at two temperatures ( $5 \mathrm{~K}$ : dashed line; $30 \mathrm{~K}$ : dotted line).

\section{APPENDIX C: BROADENING INDUCED BY THE DISPLACEMENTS AND OSCILLATIONS OF THE QUANTUM DOTS IN THE JUNCTION}

In typical STS experiments of isolated QDs, the QD is linked to the substrate, for example, by molecules, ${ }^{11}$ which mechanically can be represented by some spring of stiffness $k_{s}$. When the system is biased, there is an important electric field between the tip and the substrate and, therefore, when an electron is injected into the QD, there is an electrostatic force that induces a displacement of the QD. The effect of the coupling between the mechanical vibrations of a quantum dot placed between two leads has recently received considerable interest $\mathrm{t}^{52-55}$ in the framework of the development of the nanoelectromechanics. ${ }^{56}$ Here, we discuss how such a coupling could lead to a Gaussian broadening of the STS peaks.

We consider the system depicted in Fig. 10. We define by $x_{1}$ and $x_{2}$ the distance of the QD to the substrate and the tip, respectively. At a given bias $V, x_{1}+x_{2}$ is equal to a constant

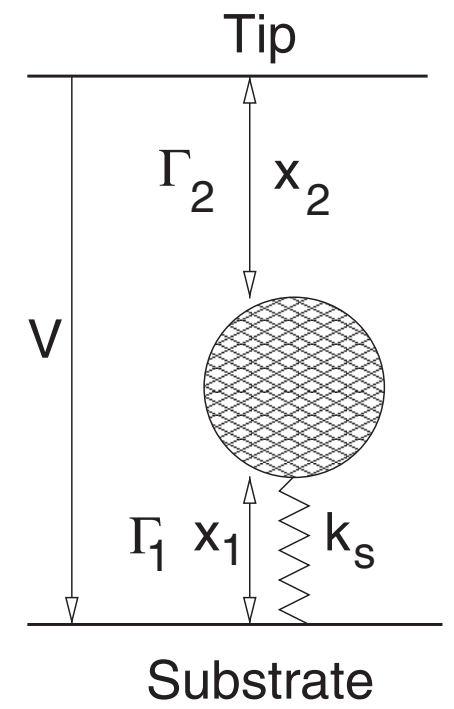

FIG. 10. Simple model of the tip-QD-substrate system in which the QD is linked to the substrate by a spring of stiffness $k_{s}$.
$X$. The total energy of the QD with a charge $q$ (in units of $e$ ) is given by $21,22,57$

$$
E=\eta e V q+\frac{1}{2} U q^{2}+\frac{1}{2} k_{s}\left(x_{1}-x_{1}^{0}\right)^{2},
$$

where $U$ is the charging energy defined above, $\eta V$ is the potential in the QD $(0 \leq \eta \leq 1)$, and $x_{1}^{0}$ is the equilibrium QD-substrate distance when $q=0$. We consider now the case where the QD is charged by one electron $(q=-1)$ and we make a Taylor expansion of $E$ around $x_{1}^{0}$,

$$
E=-\eta e \mathrm{~V}+\frac{U}{2}+B\left(x_{1}-x_{1}^{0}\right)+\frac{1}{2} k_{s}\left(x_{1}-x_{1}^{0}\right)^{2},
$$

where $B=\frac{\partial}{\partial x_{1}}[-\eta e V+U / 2]$. Minimizing this total energy with respect to $x_{1}$, we calculate the relaxation energy $\lambda_{s}$ $=B^{2} /\left(2 k_{s}\right)$ as the difference between the energies in the neutral and charged situations. To estimate $B$, we use a simple model in which the double junction of Fig. 10 is described by two capacitances, $C_{1}$ and $C_{2}$, which depend on the distances $x_{1}$ and $x_{2}$ such as $C_{1}=C_{1}^{0} x_{1}^{0} / x_{1}$ and $C_{2}=C_{2}^{0} x_{2}^{0} / x_{2}$. By using $U=e^{2} /\left(C_{1}+C_{2}\right), \eta=C_{1} /\left(C_{1}+C_{2}\right),{ }^{21,22,57}$ and $x_{1}+x_{2}=X$, we obtain

$$
B=\frac{e V C_{1}^{0} C_{2}^{0}}{\left(C_{1}^{0}+C_{2}^{0}\right)^{2}}\left[\frac{1}{x_{1}^{0}}+\frac{1}{x_{2}^{0}}\right]-\frac{e^{2}}{2\left(C_{1}^{0}+C_{2}^{0}\right)^{2}}\left[\frac{C_{2}^{0}}{x_{2}^{0}}-\frac{C_{1}^{0}}{x_{1}^{0}}\right] .
$$

With $V \approx 1 \mathrm{~V}$, where $C_{1}$ and $C_{2}$ are in the range of $1 \mathrm{aF}^{22}$ the second term in Eq. (C3) can be neglected. Using the values derived in Ref. 22 from STS spectra, $C_{1}^{0}=0.95 \mathrm{aF}$, $C_{2}^{0}=0.29 \mathrm{aF}, x_{1}^{0}=0.5 \mathrm{~nm}$, and $x_{2}^{0}=0.3 \mathrm{~nm}$, we obtain $B$ $\approx 1 \mathrm{eV} / \mathrm{nm}$.

The evaluation of the spring stiffness $k_{s}$ is obviously more difficult because it requires us to know the nature of the contact between the QD and the substrate. In the following, we estimate $k_{s}$ from the Young modulus $E_{Y}$ measured on a octadecyltriethoxysilane monolayer on mica. ${ }^{58}$ When a pressure $P$ is applied on the layer, the elastic energy per unit of volume is $P^{2} /\left(2 E_{Y}\right)$. If we define by $S_{0}$ the area of contact between the QD and the monolayer, the elastic energy in the monolayer under contact is $S_{0} x_{0} P^{2} /\left(2 E_{Y}\right)$, where $x_{0}$ is the thickness of the monolayer. The applied force at the surface is $P S_{0}$ which is also equal to $k_{s} \Delta x$ if we simulate the system by a spring and if $\Delta x$ is the displacement of the QD. Using the fact that the elastic energy in the spring is given by $\frac{1}{2} k_{s} \Delta x^{2}$, we deduce that $k_{s}=S_{0} E_{Y} / x_{0}$. With $E_{Y} \approx 0.3 \mathrm{GPa}$, ${ }^{58}$ $S_{0}=0.1 S_{\mathrm{dot}}$, where $S_{\mathrm{dot}}=4 \pi R^{2}$ is the surface of the dot, and $x_{0}=0.5 \mathrm{~nm}$, we obtain $k_{s} \approx 19 \mathrm{eV} / \mathrm{nm}^{2}$ for $R=2 \mathrm{~nm}$.

With $B \approx 1 \mathrm{eV} / \mathrm{nm}$ and $k_{s} \approx 19 \mathrm{eV} / \mathrm{nm}^{2}$, we get a relaxation energy $\lambda_{s} \approx 26 \mathrm{meV}$, which is far from being negligible. With a mass of the QD given by $M=4 \pi R^{3} \rho / 3$, where $\rho=5.81 \mathrm{~g} / \mathrm{cm}^{3}$ is the density of CdSe, the vibration energy of the oscillator is $\hbar \sqrt{k_{s} / M} \approx 8 \times 10^{-5} \mathrm{eV}$. Thus, while we are in the classical regime $(\hbar \omega \ll k T)$, the line shape is a Gaussian with a FWHM of $\approx 11 \mathrm{meV}$ at $5 \mathrm{~K}$. The frequency of the oscillator $\left(\approx 2 \times 10^{10} \mathrm{~Hz}\right)$ is higher than the tunneling rate of $6 \times 10^{8} \mathrm{~Hz}$ for $I=0.1 \mathrm{nA}$, which means that the system is not in a shuttling regime. ${ }^{56}$ 
The FWHM of $\approx 11 \mathrm{meV}$ at $5 \mathrm{~K}$ that we estimate shows that the nano-oscillation of the QD could possibly be involved in the broadening of the STS spectra. However, in that case, the FWHM should strongly depend on the tipsample distance and on the nature of the substrate, which is not the case in our experiments. We conclude that the relaxation energy due to the mechanical displacement of the QD in the junction is smaller than in our estimation, probably due to a higher stiffness of the QD-substrate link.

The model presented in this appendix is valid only if the amplitude of the oscillations is small compared to the tip-QD and QD-substrate distances. Using previous numbers, we deduce a displacement of the QD after charging $\Delta x=B / k_{s}$ $\approx 0.05 \mathrm{~nm}$, which is small compared to $x_{1}^{0}$ and $x_{2}^{0}$, justifying our approximations. However, even if $\Delta x$ is small, the variations of the tunneling rates with the displacements cannot be totally neglected. In the following, we discuss the influence of these variations on the line shape and we show that the main conclusions of this section remain valid.

We have already discussed that in the limit $k T \gg \hbar \omega$, the line shape is a Gaussian of the form $G(E)$ $\propto \exp \left[-E^{2} /(4 \lambda k T)\right]$. This expression can be classically obtained by considering the transition energy between two oscillators: the first one centered at $x_{1}=x_{1}^{0}$ (neutral QD) and the second one centered at $x_{1}=x_{1}^{0}-B / k_{s}$. In this limit, $G(E)$ is given by (with $\left.\lambda=\lambda_{s}=B^{2} / 2 k_{s}\right)^{20}$

$$
G(E) \propto \int_{-\infty}^{+\infty} \delta(E-B X) e^{-k_{s} X^{2} / 2 k T} d X,
$$

where $X=x_{1}-x_{1}^{0}, B X$ is the difference in energy between the two oscillators at a given $X$ (within a constant without importance here), and the exponential is the Boltzmann probability to have a kinetic energy $k_{s} X^{2} / 2$. This calculation supposes that the transition rate does not depend on $X$. However, in the case of a tunneling process, the tunneling rate exponentially depends on $X$, like $\exp (-\alpha X)$, where $\alpha \approx 2.3 \AA^{-1}$ (if the current decreases by a factor 10 for a $1 \AA$ increment of the tip-QD distance). Taking this effect into account, the line shape becomes

$$
G(E) \propto \int_{-\infty}^{+\infty} \delta(E-B X) e^{-\alpha X} e^{-k_{s} X^{2} / 2 k T} d X
$$

which leads to

$$
G(E) \propto e^{\alpha E / B} e^{-E^{2} /\left(4 \lambda_{s} k T\right)} \propto e^{-\left(E-E_{0}\right)^{2} /\left(4 \lambda_{s} k T\right)},
$$

where $E_{0}=2 \alpha \lambda_{s} k T / B$. Thus, we recover a Gaussian characterized by the same width but just shifted in energy. By using $\lambda_{s} \approx 26 \mathrm{meV}$, we predict a shift $E_{0} \approx 10 \mathrm{meV}$.

\section{APPENDIX D: TEMPERATURE OF THE QUANTUM DOT AND THERMAL DISSIPATION DUE TO ELECTRON TRANSPORT}

In tunneling experiments, each time an electron flows through the system, some part of its energy is released into the quantum dot by coupling to local vibrational (phonon) modes. On average, this energy is of the order of $\lambda$, which is the reorganization (or relaxation) energy. Thus, in a station-

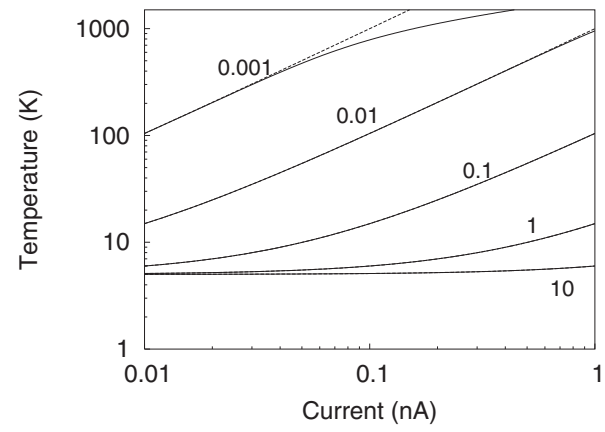

FIG. 11. Effective temperature in a quantum dot $($ size $=4 \mathrm{~nm})$ versus the tunneling current $I$ plotted for different values of the interface thermal conductance $G$ in $\mathrm{MW} \mathrm{m}^{-2} \mathrm{~K}^{-1}$. The dashed lines represent the results obtained when the dissipation through blackbody radiation is neglected. All the curves reach the value $T=T_{\text {out }}$ $=5 \mathrm{~K}$ for $I=0$.

ary regime, there is an important heat flow into the QD. The heat is transferred to the surrounding of the quantum dot, i.e., to the substrate and to the tip. The question is, however, on how far the temperature of the QD exceeds the temperature applied to the substrate. In STM experiments, the coupling between the nanostructure and its environment is weak and the heat dissipation is limited by the thermal conductance of the interfaces. ${ }^{59}$ While the temperature of the environment is kept at a constant value, $T_{\text {out }}$, the electrons and the phonons in the quantum dot can reach a local equilibrium characterized by an effective temperature $T$. It has been shown that for sufficiently small particles, the thermal conductance $G$ per unit area of the particle-environment interface plays an important role in the thermal decay of a particle heated by a laser pulse. ${ }^{59,60}$ For colloidal metal particles in fluids, values of $G$ between 5 and $130 \mathrm{MW} \mathrm{m}^{-2} \mathrm{~K}^{-1}$ are reported ${ }^{59}$ and thus probably provide upper values for situations corresponding to STM experiments. On this basis, we can write the rate equation for the heat exchange in the quantum dot,

$$
\frac{d Q}{d t}=\left(\frac{I}{e}\right) \lambda-G S_{c}\left[T(t)-T_{\text {out }}\right]-\sigma S_{\text {dot }} T(t)^{4},
$$

where I/e is the tunneling rate from the tip into the dot and $S_{c}$ is the contact area across which heat is transferred from the dot to the environment. The last term in Eq. (D1) describes the heat dissipation by black-body radiation to the free space, where $\sigma=5.67 \times 10^{-8} \mathrm{~W} \mathrm{~m}^{-2} \mathrm{~K}^{-4}$ is the Stefan constant. The temperature of the dot under steady state condition $(d Q / d t=0)$ becomes

$$
T=T_{\text {out }}+\frac{I \lambda}{e G S_{c}}
$$

when the black-body radiation can be neglected, otherwise Eq. (D1) must be numerically solved. Figure 11 presents the variation of $T$ with the current $I$ for different values of $G$ from $10^{1}$ to $10^{-3} \mathrm{MW} \mathrm{m} \mathrm{m}^{-2} \mathrm{~K}^{-1}$. We have considered a typical situation where $R=2 \mathrm{~nm}, \lambda=50 \mathrm{meV}$, and $T_{\text {out }}=5 \mathrm{~K}$. We have also assumed a small contact area $S_{c}=0.1 S_{\text {dot }}$. Figure 11 shows that the black-body radiation is negligible except at high temperatures $(\geq 300 \mathrm{~K})$ which can be 
reached for small values of $G\left(\ll 1 \mathrm{MW} \mathrm{m}^{-2} \mathrm{~K}^{-1}\right)$. For $G$ $\gtrsim 1 \mathrm{MW} \mathrm{m}^{-2} \mathrm{~K}^{-1}$, i.e., for a thermal conductance at the contacts comparable to the values reported for the solidfluid interface, the temperature rise is below $5 \mathrm{~K}$. However,
Fig. 11 shows that the nature of the coupling between the dot and the substrate plays an important role in the heat dissipation, and that dissipative heating might occur in some cases.
${ }^{1}$ J. Repp, G. Meyer, F. E. Olsson, and M. Persson, Science 305, 493 (2004).

${ }^{2}$ F. E. Olsson, M. Persson, A. G. Borisov, J. P. Gauyacq, J. Lagoute, and S. Folsch, Phys. Rev. Lett. 93, 206803 (2004).

${ }^{3}$ A. J. Heinrich, J. A. Gupta, C. P. Lutz, and D. M. Eigler, Science 306, 466 (2004).

${ }^{4}$ M. Berthe, A. Urbieta, L. Perdigão, B. Grandidier, D. Deresmes, C. Delerue, D. Stiévenard, R. Rurali, N. Lorente, L. Magaud, and P. Ordejón, Phys. Rev. Lett. 97, 206801 (2006).

${ }^{5}$ J. Repp, G. Meyer, S. Paavilainen, F. E. Olsson, and M. Persson, Phys. Rev. Lett. 95, 225503 (2005).

${ }^{6}$ J. Repp, G. Meyer, S. M. Stojkovic, A. Gourdon, and C. Joachim, Phys. Rev. Lett. 94, 026803 (2005).

${ }^{7}$ X. H. Qiu, G. V. Nazin, and W. Ho, Phys. Rev. Lett. 92, 206102 (2004).

${ }^{8}$ S. W. Wu, G. V. Nazin, X. Chen, X. H. Qiu, and W. Ho, Phys. Rev. Lett. 93, 236802 (2004).

${ }^{9}$ X. Lu, M. Grobis, K. H. Khoo, S. G. Louie, and M. F. Crommie, Phys. Rev. Lett. 90, 096802 (2003).

${ }^{10}$ N. A. Pradhan, N. Liu, C. Silien, and W. Ho, Phys. Rev. Lett. 94, 076801 (2005).

${ }^{11}$ U. Banin, Y. Cao, D. Katz, and O. Millo, Nature (London) 400, 542 (1999).

${ }^{12}$ L. Jdira, P. Liljeroth, E. Stoffels, D. Vanmaekelbergh, and S. Speller, Phys. Rev. B 73, 115305 (2006).

${ }^{13}$ B. Grandidier, Y. M. Niquet, B. Legrand, J. P. Nys, C. Priester, D. Stiévenard, J. M. Gérard, and V. Thierry-Mieg, Phys. Rev. Lett. 85, 1068 (2000).

${ }^{14}$ T. Maltezopoulos, A. Bolz, C. Meyer, C. Heyn, W. Hansen, M. Morgenstern, and R. Wiesendanger, Phys. Rev. Lett. 91, 196804 (2003).

${ }^{15}$ X. H. Qiu, G. V. Nazin, and W. Ho, Science 299, 542 (2003).

${ }^{16}$ J. Zhao, C. Zeng, X. Cheng, K. Wang, G. Wang, J. Yang, J. G. Hou, and Q. Zhu, Phys. Rev. Lett. 95, 045502 (2005).

${ }^{17}$ E. Bakkers, Z. Hens, A. Zunger, A. Franceschetti, L. Kouwenhoven, L. Gurevich, and D. Vanmaekelbergh, Nano Lett. 1, 551 (2001).

${ }^{18}$ O. Millo, D. Katz, Y. W. Cao, and U. Banin, Phys. Rev. B 61, 16773 (2000).

${ }^{19}$ N. A. Pradhan, N. Liu, and W. Ho, J. Phys. Chem. B 109, 8513 (2005).

${ }^{20} \mathrm{C}$. Delerue and M. Lannoo, Nanostructures: Theory and Modeling (Springer-Verlag, Berlin, 2004).

${ }^{21}$ Y. M. Niquet, C. Delerue, M. Lannoo, and G. Allan, Phys. Rev. B 64, 113305 (2001).

${ }^{22}$ Y. M. Niquet, C. Delerue, G. Allan, and M. Lannoo, Phys. Rev. B 65, 165334 (2002).

${ }^{23}$ J. M. An, A. Franceschetti, S. V. Dudiy, and A. Zunger, Nano Lett. 6, 2728 (2006).

${ }^{24}$ C. de Mello Donega, S. G. Hickey, S. F. Wuister, D. Vanmaekelbergh, and A. Meijerink, J. Phys. Chem. B 107, 489 (2003).
${ }^{25}$ P. Liljeroth, L. Jdira, K. Overgaag, B. Grandidier, S. Speller, and D. Vanmaekelbergh, Phys. Chem. Chem. Phys. 8, 3845 (2006).

${ }^{26}$ J. G. A. Dubois, E. N. G. Verheijen, J. W. Gerritsen, and H. van Kempen, Phys. Rev. B 48, 11260 (1993).

${ }^{27}$ P. Liljeroth, K. Overgaag, A. Urbieta, B. Grandidier, S. G. Hickey, and D. Vanmaekelbergh, Phys. Rev. Lett. 97, 096803 (2006).

${ }^{28}$ D. Steiner, A. Aharoni, U. Banin, and O. Millo, Nano Lett. 6, 2201 (2006).

${ }^{29}$ C. Delerue, G. Allan, and Y. M. Niquet, Phys. Rev. B 72, 195316 (2005).

${ }^{30}$ N. S. Wingreen, K. W. Jacobsen, and J. W. Wilkins, Phys. Rev. B 40, 11834 (1989).

${ }^{31}$ U. Lundin and R. H. McKenzie, Phys. Rev. B 66, 075303 (2002).

${ }^{32}$ K. Huang and A. Rhys, Proc. R. Soc. London, Ser. A 204, 406 (1950).

${ }^{33}$ J. Bourgoin and M. Lannoo, Point Defects in Semiconductors II, Springer Series in Solid-State Sciences Vol. 35 (Springer-Verlag, Berlin, 1983).

${ }^{34}$ E. Lampin, C. Delerue, M. Lannoo, and G. Allan, Phys. Rev. B 58, 12044 (1998).

${ }^{35}$ M. Lax, J. Chem. Phys. 20, 1752 (1952).

${ }^{36}$ T. H. Keil, Phys. Rev. 140, A601 (1965).

${ }^{37}$ J. S. Pan and H. B. Pan, Phys. Status Solidi B 148, 129 (1988).

${ }^{38}$ J. C. Marini, B. Stebe, and E. Kartheuser, Phys. Rev. B 50, 14302 (1994).

${ }^{39}$ K. Oshiro, K. Akai, and M. Matsuura, Phys. Rev. B 58, 7986 (1998).

${ }^{40}$ D. V. Melnikov and W. B. Fowler, Phys. Rev. B 63, 165302 (2001).

${ }^{41}$ M. Nirmal, B. O. Dabbousi, M. G. Bawendi, J. J. Macklin, J. K. Trautman, T. D. Harris, and L. E. Brus, Nature (London) 383, 802 (1996)

${ }^{42}$ R. G. Neuhauser, K. T. Shimizu, W. K. Woo, S. A. Empedocles, and M. G. Bawendi, Phys. Rev. Lett. 85, 3301 (2000).

${ }^{43}$ B. R. Fisher, H.-J. Eisler, N. E. Stott, and M. G. Bawendi, J. Phys. Chem. B 108, 143 (2004).

${ }^{44}$ J. Müller, J. M. Lupton, A. L. Rogach, J. Feldmann, D. V. Talapin, and H. Weller, Phys. Rev. Lett. 93, 167402 (2004).

${ }^{45}$ S. A. Empedocles and M. G. Bawendi, Science 278, 2114 (1997).

${ }^{46}$ T. Takagahara, Phys. Rev. Lett. 71, 3577 (1993).

${ }^{47}$ T. Takagahara, J. Lumin. 70, 129 (1996).

${ }^{48}$ E. Martin, C. Delerue, G. Allan, and M. Lannoo, Phys. Rev. B 50, 18258 (1994).

${ }^{49}$ A. Franceschetti and S. T. Pantelides, Phys. Rev. B 68, 033313 (2003).

${ }^{50}$ R. A. Marcus, Annu. Rev. Phys. Chem. 16, 155 (1965).

${ }^{51}$ L. E. Brus, Phys. Rev. B 53, 4649 (1996).

${ }^{52}$ H. Park, J. Park, A. K. L. Lim, E. H. Anderson, A. P. Alivisatos, 
and P. L. McEuen, Nature (London) 407, 57 (2000).

${ }^{53}$ D. Boese and H. Schoeller, Europhys. Lett. 54, 668 (2001).

${ }^{54}$ D. Fedorets, L. Y. Gorelik, R. I. Shekhter, and M. Jonson, Europhys. Lett. 58, 99 (2002).

${ }^{55}$ K. D. McCarthy, N. Prokof'ev, and M. T. Tuominen, Phys. Rev. B 67, 245415 (2003).

${ }^{56}$ R. I. Shekhter, Y. Galperin, L. Y. Gorelik, A. Isacsson, and M. Jonson, J. Phys.: Condens. Matter 15, R441 (2003).
${ }^{57}$ R. I. Shekhter, Zh. Eksp. Teor. Fiz. 63, 1410 (1972) [Sov. Phys. JETP 36, 747 (1973)]; I. O. Kulik and R. I. Shekhter, Zh. Eksp. Teor. Fiz. 68, 623 (1975) [Sov. Phys. JETP 41, 308 (1975)].

${ }^{58}$ W. Kiridena, V. Jain, P. K. Kuo, and G. Liu, Surf. Interface Anal. 25, 383 (1997).

${ }^{59}$ O. M. Wilson, X. Hu, D. G. Cahill, and P. V. Braun, Phys. Rev. B 66, 224301 (2002).

${ }^{60}$ M. Hu and G. V. Hartland, J. Phys. Chem. B 106, 7029 (2002). 